\title{
El agotamiento de los recursos pesqueros y la emigración: el caso de la laguna de Tecomate, Guerrero, México
}

The depletion of fish stocks and migration: the case of Tecomate Lagoon, Guerrero, Mexico

O esgotamento dos recursos haliêuticos e de migração: o caso do Lago Tecomate, Guerrero, México

Salvador Villerías Salinas

Universidad Autónoma de Guerrero, México svilleríass@gmail.com

Neftalí García Castro CONACYT-Universidad Autónoma de Guerrero, México neftaligc@hotmail.com

Pedro Vidal Tello Almaguer Universidad Autónoma de Guerrero, México pvidalt@hotmail.com

Práxedes Muñoz Sánchez Universidad Católica de Murcia, España praxedesm@gmail.com

\section{Resumen}

Las lagunas costeras son importantes por ser refugios de especies acuáticas, terrestres y dar alimento a la población humana asentada en sus márgenes. El deterioro ambiental ha hecho que disminuyan los recursos pesqueros $\mathrm{y}$, por tanto, ha afectado a las poblaciones ubicadas en el margen de la laguna de Tecomate, las cuales tienen un precario desarrollo socioeconómico, al igual que muchas otras localidades de México. Los hallazgos de esta investigación tienen que ver con la escasez de los recursos pesqueros ocasionados por la poca profundidad del lago, su alta concentración en sal y temperatura en la época estival, y lo contrario en la estación de lluvias. El ecosistema sufre radicales y opuestas alteraciones ambientales a lo largo del año, lo que afecta la 
biodiversidad íctica, esencial para la subsistencia de la población asentada en la ribera de la laguna. Asimismo, los escasos recursos pesqueros ocasionan que la población joven migre en busca de trabajo para mejorar su nivel de vida. Se requieren estrategias de desarrollo desde un enfoque sustentable y la intervención de los actores locales para planificar el uso y conservación de los recursos pesqueros.

Palabras clave: agotamiento, recurso pesquero, migración, laguna de Tecomate.

\section{Abstract}

The coastal lagoons are important as refuges for aquatic and terrestrial species and provide food to human population settled in its shores. Environmental degradation has decreased fish stocks and, thereforehas affected populations located on the Tecomate lagoon shores, which have a precarious socio-economic development, just like many other towns in Mexico. The findings of this research have to do with the shortage of fish stocks due to the shallow depth of the Lake, its high salt concentration and temperature in the summer season, and otherwise in the rainy season. The ecosystem suffers radicals and opposing environmental alterations throughout the year, which affects the biodiversity of fish, essential for the subsistence of the population settled on the banks of the lagoon. Also scarce fishery resources cause young people to migrate in search of work to improve their standard of living. Strategies of development from a sustainable approach and the involvement of local actors is required to plan the use and conservation of fishery resources.

Key Words: depletion, exhaustion, fish stocks, fishery resources, migration, Tecomate Lagoon.

\section{Resumo}

As lagoas costeiras são importantes por ser um refúgio de espécies aquáticas, terrestres e fornecer alimentos para a população humana vivendo em suas margens. A degradação ambiental tem causado o declínio dos recursos haliêuticos e, populações, portanto, tem afetado na margem da lagoa Tecomate, que têm um desenvolvimento sócio-económico pobres, como muitas outras cidades no México. Os resultados desta pesquisa têm a ver com a escassez dos recursos da pesca causados pela superficialidade do lago, alta concentração de sal e da temperatura no verão, eo oposto na estação chuvosa. O ecossistema sofre mudanças radicais e opostas ambientais ao longo 
do ano, afetando a biodiversidade de peixes, essenciais para a sobrevivência da população que vive nas margens da lagoa. Também escassos recursos haliêuticos que causam os jovens a migrar em busca de trabalho para melhorar seu padrão de vida. estratégias de desenvolvimento são necessários a partir de uma abordagem sustentável e a participação dos agentes locais para planejar o uso e conservação dos recursos haliêuticos.

Palavras-chave: exaustão, os recursos haliêuticos, migração, Tecomate lagoa.

Fecha recepción: Enero 2016

Fecha aceptación: Junio 2016

\section{Introducción}

Las acciones emprendidas por el gobierno federal para el desarrollo y fortalecimiento de los recursos hídricos en los distritos de riego no fueron sustentadas en estudios de impacto ambiental, sino en decisiones de los gobernantes en turno tomadas a partir de su visión de desarrollo y su particular forma de gobernar. Al momento de hacer efectivas sus iniciativas sólo consideraron el número de población que se vería "beneficiada", en contraposición con el número de la "no beneficiada", sin detenerse a pensar en los efectos a corto y largo plazo que podrían ocasionar en el medio ambiente, sobre todo en el caso de la laguna de Tecomate, con la construcción de la Presa Revolución Mexicana a principios de la década de los años ochenta del siglo pasado. Las comunidades de Tecomate Pesquerías, Cerro la Pesquería y Las Ramaditas, ubicadas a la vera de la laguna, han sido afectadas en la pesca, su actividad principal, por lo que han tenido que desarrollar diferentes estrategias para sobrevivir. Además de realizar actividades de agricultura, como la siembra temporal de maíz, o el cuidado de huertas de coco, han emigrado. Estos procesos migratorios se reflejan en el número de su población, pues si bien durante el periodo de 1970 a 2010 el saldo poblacional neto era positivo, en una de estas comunidades fue negativo. A primera vista parece que la construcción de la presa y sus repercusiones en el medio ambiente provocaron la migración de la población, pero si se analiza con más detalle la situación se puede observar que se conjuntan factores ligados a la crisis económica de los años ochenta y a las facilidades que en ese entonces había para migrar de manera temporal. Todo esto facilitó que los migrantes pudieran 
estar presentes de manera periódica, aumentando así la población y, posteriormente, con la ayuda de las remesas, que se desarrollaran las comunidades.

En la actualidad, el corte de suministro de agua dulce a la laguna por el río Nexpa, produce cambios drásticos en las condiciones del régimen hidrológico lagunar y en los niveles de captura de las especies nativas. La cercanía con la cabecera municipal de San Marcos, así como con el puerto de Acapulco, permite a los pobladores desarrollar, además de las actividades agrícolas mencionadas, actividades económicas que les garantizan la obtención del sustento por medio de empleos temporales o definitivos, así como de las remesas que reciben de la población migrante y la ayuda que reciben de los programas gubernamentales.

\section{Metodología}

El trabajo de investigación se realizó en tres localidades ubicadas a la vera de la Laguna de Tecomate, la cual tiene una extensión de $28 \mathrm{Km}^{2}$, localizada en los $16^{\circ} 41^{\prime} 28^{\prime}$ ' de latitud norte y 99 19' 40', de longitud oeste, a 11 km de San Marcos, cabecera municipal del municipio del mismo nombre. Las localidades Tecomate Pesquerías, Cerro la Pesquería y Las Ramaditas se encuentran en el borde lagunar (figura 1), cuyos asentamientos tienen un vínculo muy estrecho con la laguna porque les provee de recursos (peces y crustáceos) para subsistir, aunque también se dedican a actividades agrícolas.

Con el fin de observar la estructura y organización de las comunidades, en particular el desarrollo de la actividad pesquera, se llevaron a cabo recorridos en el entorno lagunar y en las comunidades seleccionadas; además, se aplicaron encuestas para conocer el tipo de captura y los montos aproximados de las mismas, y entrevistas semiestructuradas para conocer de viva voz de los actores las tendencias y comportamiento de la producción, así como de la población. 
Figura 1. Laguna de Tecomate: localización.

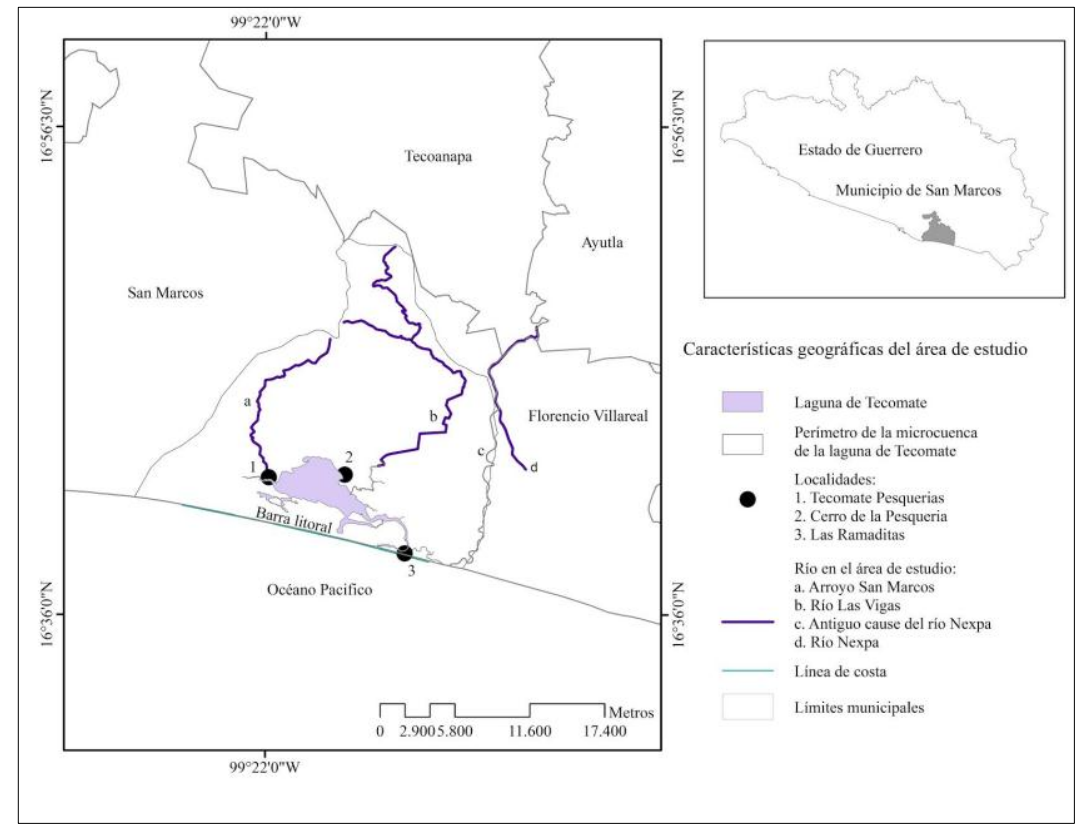

Fuente: trabajo de campo e INEGI (2016).

\section{Resultados}

La actividad pesquera genera producción económica, social, alimentaria y cultural, además crea empleos y da sustento a varias familias que viven en los alrededores de la laguna de Tecomate; sin embargo, la pesca ha dejado de ser su principal actividad económica.

En la laguna de Tecomate se identificaron cuatro especies: lisa (Mugil sp), malacapa (Diapterus peruvianos), bandera (Ariidae), pijolín (Centropomus robalito), tilapia y jaiba. El valor comercial de la pesca está en función de la oferta y demanda, además de las temporadas vacacionales (semana santa, diciembre y puentes) (tabla 1). A pesar de que existen cooperativas pesqueras no llevan registro alguno de las capturas, lo que hizo difícil la estimación de su volumen. En entrevista, los pescadores manifestaron que después de la construcción de la presa Revolución Mexicana y de haber cerrado el paso del río Nexpa a la laguna, la pesca ha decaído y es muy escasa. Lo poco que pescan sólo ayuda a complementar sus ingresos. 
Tabla 1. Laguna de Tecomate: especies y valor de la captura, 2014.

\begin{tabular}{|l|l|c|}
\hline $\begin{array}{l}\text { Nombre } \\
\text { común }\end{array}$ & Nombre científico & $\begin{array}{l}\text { Valor de la captura por } \\
\text { kilogramo (pesos) }\end{array}$ \\
\hline Lisa & Mugil cephalus (Linnaeus, 17589 & 20.00 \\
\hline Malacapa & Diapterus peruvianos (Cuvier, 1830) & 25.00 \\
\hline Cuatete & Ariopsis guatemalensis (Günther, 1864) & 30.00 \\
\hline Pijolín & Centropomus robalito (Jordan y Gilbert, 1882) & 40.00 \\
\hline Jaiba & Callinectes arcuatus (Orday, 1863) & 25.00 \\
\hline Camarón & Penaeus vannamei (Boone, 1931) & 80.00 \\
\hline Langostino & Macrobranchium americanum (Bowman y Abele, 1882) & 60.00 \\
\hline
\end{tabular}

Fuente: trabajo de campo (2014).

Los pobladores consideran que la laguna de Tecomate se está muriendo porque tiene poca agua, además de que en temporada de secas el agua se calienta demasiado y su nivel baja mucho.

\section{Organización para la pesca}

La pesca en la laguna como un "área común de captura" es llevada a cabo por organizaciones ubicadas en Tecomate Pesquerías, Cerro de la Pesquería y Las Ramaditas. De una población que ascendía a 884 habitantes en el año 2010 en las tres localidades, 274 se dedican a la pesca (99.63 $\%$ de la PEA). La pesca se distribuye de la manera siguiente: $60 \%$ se ubica en Tecomate Pesquerías, $26 \%$ en Cerro de la Pesquería y $14 \%$ en Las Ramaditas. Esta importante población de pescadores es muy alta si se compara con la superficie del cuerpo de agua de la laguna (1:0.10 $\mathrm{km}^{2}$ ); existe una gran presión pesquera, otra probable causa del agotamiento del recurso pesquero.

Los pescadores son nativos de las tres localidades y los que han emigrado son oriundos de comunidades circunvecinas; se deduce entonces que las personas dedicadas a la pesca tienen un gran arraigo e identificación con su localidad. Por otro lado, en la microrregión persiste la conducta individualista en los pescadores ribereños, quienes se muestran indiferentes al desarrollo económico y social de sus localidades y, en consecuencia, apatía para asociarse y enfrentar de manera conjunta, organizada y eficiente la función productiva que desempeñan. Este escenario se presenta en las entidades pesqueras del Pacífico sur, como mencionan Villaseñor y 
García (1990) y por tanto en la costa de Jalisco, donde encontraron muchas cooperativas desorganizadas y con deficiencias en respecto al registro del producto pesquero.

No obstante, los pescadores de esta microrregión no se oponen totalmente al cambio técnico. Aparentemente, su escaso interés a cambiar se debe a la falta de conocimiento respecto a las oportunidades o posibilidades de acceder a los programas de apoyo implementados por el gobierno, o a la escasez de recursos financieros u otras reservas, requisitos previos para aceptar el riesgo de incursionar en algo nuevo. También está claro el desinterés de los tres niveles de gobierno (federal, estatal y municipal), por resolver o paliar la situación de deterioro socioambiental en la laguna. Sólo en campañas electorales se pronuncian promesas de solución para este problema.

\section{El comportamiento de la población}

Además de analizar el impacto en las condiciones naturales de la laguna, la reproducción de su ecosistema y la actividad pesquera, fue importante considerar el comportamiento de la población en las comunidades que la circundan. Para ello se analizó el periodo 1970 - 2010, cuando hubo cuatro censos y dos conteos de población por parte del Instituto Nacional de Estadística Geografía e Informática (tabla 2).

En 1970 no se tenían datos de población de la comunidad Las Ramaditas, de lo que se deduce que su tamaño no era significativo o aún no se había fundado, mientras que las comunidades de Tecomate Pesquerías y Cerro de la Pesquería ya figuraban en los censos de población.

Tabla 2. Laguna de Tecomate: población total de las comunidades, 1970-2010.

\begin{tabular}{lccccccc}
\hline \multicolumn{1}{c}{ Localidades } & \multicolumn{7}{c}{ Años } \\
\cline { 2 - 8 } & 1970 & 1980 & 1990 & 1995 & 2000 & 2005 & 2010 \\
\hline Tecomate Pesquerías & 353 & 485 & 277 & 371 & 527 & 472 & 578 \\
Cerro de la Pesquería & 188 & 163 & 133 & 159 & 122 & 92 & 114 \\
Las Ramaditas & & 143 & 116 & 150 & 184 & 149 & 152 \\
$\quad$ Total & 541 & 791 & 526 & 680 & 883 & 713 & 844 \\
\hline
\end{tabular}

Fuente: elaboración propia con base en información de la Secretaría de Industria y Comercio, 1973 e INEGI, 1983, 1991, 2001 y 2011. 
El comportamiento de la población en las tres comunidades era atípico debido a las variaciones abruptas en el incremento y decremento poblacional. Si no se hace un análisis adecuado se puede caer en valoraciones a priori, lo que llevaría a conclusiones inadecuadas. Es importante considerar que los estudios sobre procesos migratorios en las comunidades pesqueras de las costas de México son escasos, tanto en la vertiente del golfo como en la del Pacífico.

Al considerar el comportamiento de las tasas de crecimiento intercensal de la población, se observa que las comunidades objeto de estudio han tenido de manera alternada crecimientos en su población tanto positivos como negativos. Durante la década de 1980 a 1990, todas la comunidades presentaron tasas de crecimiento negativas, situación que se volvió a presentar en el quinquenio de 2000 a 2005; en tanto que en los quinquenios 1990 a 1995 y de 2005 a 2010, todas la comunidades presentaron tasas de crecimiento positivas (figura 2).

Figura 2. Tasa de crecimiento poblacional de las comunidades circundantes a la laguna de Tecomate.

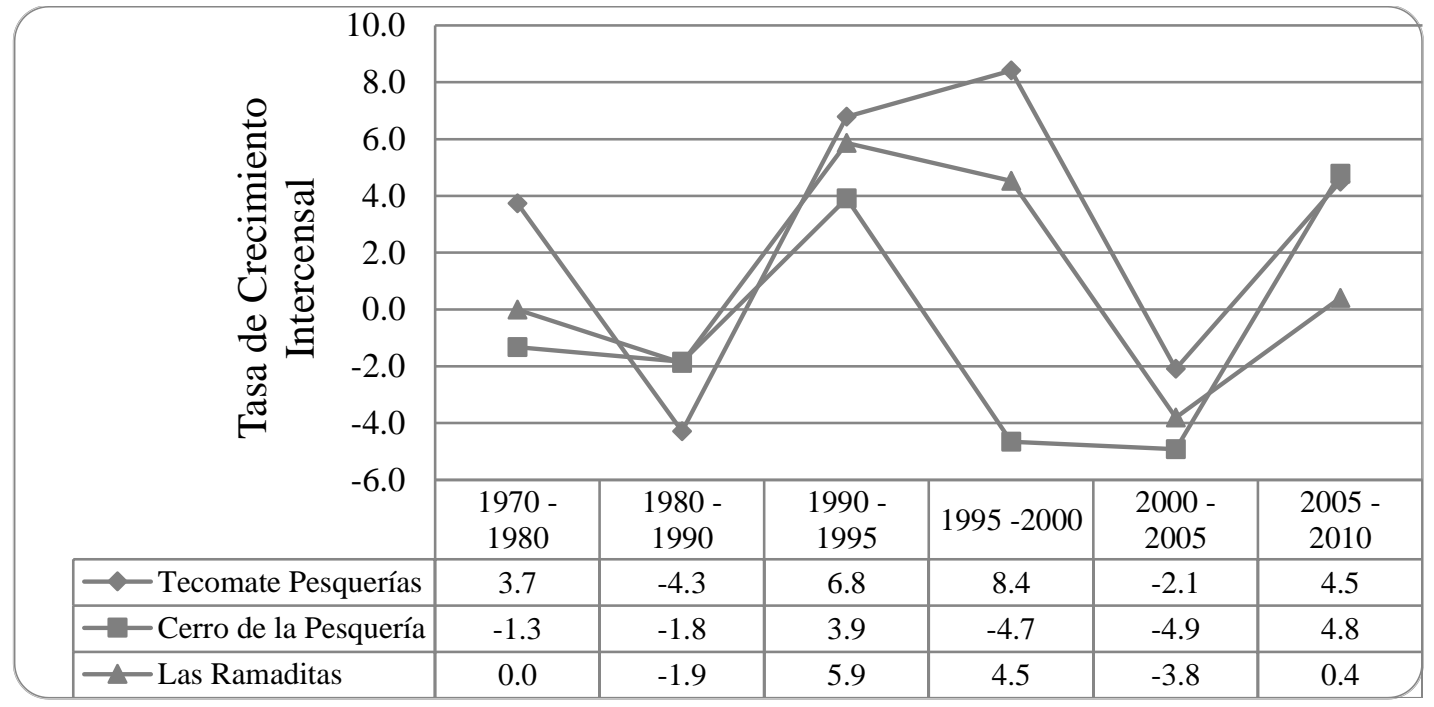

Fuente: elaboración propia con base en la tabla 1.

García et al. (2007) señalan que México en la actualidad es el primer exportador mundial de migrantes y el tercer receptor de remesas, por lo que los migrantes mexicanos es el principal grupo poblacional de extranjeros en Estados Unidos. Por su parte, Madera (2010) indica que los motivos y razones que propician la migración son: curiosidad motivada por la escucha de narraciones por parte de los habitantes de la localidad; búsqueda de ingresos ante la carencia de medios de subsistencia en la localidad o región; imposibilidad de conseguir un buen empleo o continuar con estudios superiores; acompañar al cónyuge en su migración. 
Otros puntos de interés son las catástrofes naturales, la escasez de recursos naturales y la degradación ambiental, pues pueden también generar las migraciones. Los cambios ambientales paulatinos o imprevistos ocasionan a corto o largo plazo la movilidad y desplazamiento de personas, lo que afecta las condiciones de vida y la obtención de los medios de sustento. Además, el aumento de la migración puede contribuir a una mayor degradación ambiental, pero también puede permitir hacer frente a las dificultades económicas, por lo que se vuelve una estrategia de supervivencia para quienes se desplazan.

Con base en lo señalado, se habla de migrantes por causas ambientales (Cassels et al., 2005., Black et al., 2011). Ellos son las personas o grupos de personas que, por cambios repentinos o progresivos en el medio ambiente, ven afectadas negativamente sus condiciones de vida y así se ven obligados a abandonar sus lugares de residencia habitual, ya sea temporal o permanentemente, para desplazarse a otras zonas en su país o en el extranjero.

Después de considerar las tasas de crecimiento intercensal de la población, así como la definición de migrantes por causas ambientales, se observa que las comunidades circundantes a la laguna de Tecomate, durante la década de 1980 a 1990, se redujeron en las tres localidades. La construcción de la presa Revolución Mexicana fueron y la reducción de la alimentación de agua dulce impactaron negativamente en la pesca, además dentro de un marco de crisis económica. México, igual que el resto de los países en vías de desarrollo, no escapó a las disposiciones establecidas por los organismos financieros internacionales, y para enfrentar sus crisis tuvo que adaptar su política económica al modelo económico imperante.

En consecuencia, durante la década 1980 - 1990, los cambios en la población de las comunidades objeto de estudio más que ser el resultado de la construcción de la presa y de políticas económicas ligadas al desarrollo de los distritos de riego — que afectaron las actividades pesqueras-, lo fue del impacto de la crisis económica. Además, como viajar a Estados Unidos era más fácil que ahora eso permitió la movilidad de la población, es decir, la migración recayó principalmente en los varones, quienes al visitar la comunidad solían engendrar un nuevo integrante.

En años recientes, en particular en el quinquenio 2005 - 2010, el crecimiento de la población en las comunidades de Cerro de la Pesquería y Las Ramaditas no fue muy alto. En la primera comunidad fue de 22 y en la segunda de 2 habitantes, que contrasta con la de Tecomate Pesquerías que fue de 106 (tabla 1). El incremento del número de casas habitación, así como de 
pobladores, se relaciona con la llegada de gente de otras localidades atraídas por la belleza natural del entorno, así como de la llegada de remesas por parte de los familiares de migrantes. El crecimiento de las familias hace necesaria la construcción de más viviendas.

Lo anterior, aunado a las condiciones naturales del entorno, en particular de la laguna, producto de la recuperación de los niveles de población y crecimiento del número de viviendas, va a incrementar la demanda de los servicios públicos: luz, agua entubada, drenaje y pavimentación; lo que traerá un efecto negativo al no contarse con los medios adecuados para garantizar la sustentabilidad del medio ambiente de la laguna.

Se debe tomar en cuenta también que la cercanía con la cabecera municipal permite la movilidad de los habitantes de las comunidades para desarrollar actividades complementarias o asumir un empleo definitivo en actividades ajenas a las que se desarrollan en la comunidad, lo que permite señalar que durante cuarenta años se ha presentado un proceso de movilidad por cuestiones de carácter económico más que ambiental.

\section{Discusión}

La laguna de Tecomate se ubica dentro de la región hidrológica No. 20, que comprende desde el río Papagayo hasta el río Verde, en Oaxaca. El área de estudio forma parte de la cuenca del río Nexpa. En la actualidad, la laguna de Tecomate sólo recibe aporte de una cuenca de $384 \mathrm{~km}^{2}$ y dos arroyos que drenan en forma directa; son estos los que nutren el sistema y están influidos por las características del régimen climático que prevalece en la región. Sin embargo, el sistema hidrológico lagunar está limitado por el aporte de agua dulce y agua de mar que entra.

La construcción de la presa Revolución Mexicana es resultado de las gestiones realizadas por la Liga de Comunidades Agrarias (hoy Confederación Nacional Campesina). Dicha obra hidráulica se ubica en el cauce del río Nexpa, a 1 km al noroeste de la población de El Guineo y de Las Vigas, en el municipio de San Marcos, de la región Costa Chica del estado de Guerrero. En 1984 se concluyó la construcción de la presa Revolución Mexicana (con una capacidad de 260 millones de $\mathrm{m}^{3}$ ), para generar electricidad y riego a una extensión de 14983 ha en los municipios de Florencio Villareal, Cuautepec y San Marcos, y se formó el distrito de riego 105 (SEMARNAT, s.f.). 
Una vez que entra en operación la presa y el sistema de riego, el agua del cauce principal del río Nexpa fue disminuido. El cauce del río Nexpa en la planicie costera se ubica dentro Florencio Villarreal y la poca agua que escurría fue enviada a la laguna de Chautengo, ubicada en Florencio Villarreal. La conexión hacia la laguna de Tecomate, en consecuencia, se quedó sin el principal aporte de agua dulce y su régimen de inundación se vio diezmado, al igual que las fases hidrológicas, según Bayley y Petrere (1986). Para Novoa (1986), al dejar de tener corriente el canal principal del río se propició que la laguna tendiera a mantener desde hace 32 años un nivel bajo de agua. En el verano, en los meses de junio a septiembre, la laguna recibe temporalmente una afluencia de agua dulce que supera la alimentación del resto del año, por lo que se rebasa el nivel de inundación. Los parámetros fisicoquímicos (temperatura y salinidad), además de la baja profundidad, han contribuido a la inestabilidad y al proceso de deterioro ambiental de la laguna (Villerías et al., 2016).

No se debe perder de vista que las lagunas costeras nacieron en la última glaciación, ocurrida hace aproximadamente unos 18 mil años, y que la elevación del nivel del mar ocasionó que el océano invadiera depresiones costeras, valles y deltas de ríos, generando así la actual línea litoral, que incluye bahías y entradas de mar. En las áreas donde existía aporte de agua proveniente del drenaje continental se formaron estuarios y lagunas costeras.

Una característica sobresaliente es la formación de un cordón arenoso paralelo a la costa, que revela la acumulación de arena como resultado de procesos marinos y eólicos, en formaciones de tamaño irregular en anchura, las cuales son importantes debido a que ayudan a proteger las lagunas costeras; además, en estas barras se efectúa el intercambio de agua subsuperficial y subterránea de agua dulce y marina.

Los procesos hidrológicos y geológicos locales en la laguna, así como las políticas de desarrollo de los distritos de riego a partir de la construcción de la presa, han sido factores determinantes para que este cuerpo de agua esté en un proceso de azolvamiento. Además, se debe considerar el transporte sedimentario del medio marino de norte a sur y la cercanía de la desembocadura del río Papagayo como principal afluente de sedimentos de origen continental, produciendo la formación en la laguna de una barra sedimentaria (figura 1) que separa al mar de la laguna. Su máxima anchura es de $2.9 \mathrm{~km}$ y se localiza frente a la comunidad Tecomate Pesquerías y su amplitud menor es de $1.3 \mathrm{~km}$. ubicada cerca de la comunidad de Las Ramaditas. Este proceso de 
acumulación marina (barra de la laguna) es de origen natural por efectos de las corrientes marinas marginales.

De la misma manera se debe considerar que existe una erosión del suelo como consecuencia de las actividades antropogénicas que se desarrollan en la parte alta de la cuenca, las cuales suscitan el deterioro de los recursos naturales (deterioro socio-ambiental); degradación que tiene efectos en la laguna (escasez de escurrimientos e incremento de sedimentos). En consecuencia, se afectan negativamente los recursos pesqueros que se desarrollan en dicho cuerpo de agua. Ello ha trascendido en la actividad económica de la pesca, que se emplaza en las tres localidades asentadas en el borde lagunar, por lo que es importante comentar algunos datos de la población que ahí habita.

Un factor importante que se considera en el presente análisis espacial es el de la participación de la población económicamente activa (PEA) local y la relacionada con la actividad pesquera. Cabe destacar que la PEA está muy relacionada con el producto interno bruto de cualquier región por derivar de las actividades que se desarrollan en el territorio, lo que a su vez involucra a la población que se emplea en ellas.

La economía guerrerense presenta profundos problemas estructurales: el PIB estatal está por debajo de la media nacional, hay atraso y descapitalización del campo, un alto porcentaje de la PEA ubicada en el sector informal o en actividades agropecuarias de baja productividad y un deficiente desarrollo del sector secundario. Las evidencias así lo demuestran: tres de cada cinco personas no pueden satisfacer sus necesidades básicas y, más aún, dos de cada cinco no tienen lo suficiente para comer; no tienen acceso a lo mínimo indispensable para su desarrollo y sobreviven por abajo del "límite oficial de la pobreza". En el medio rural este problema es más agudo y las expresiones más lacerantes de la pobreza extrema se presentan en los pueblos indígenas y en algunos donde los servicios básicos no existen. Cerca del 68 \% de la población de Guerrero y $46 \%$ de la población del municipio de San Marcos, donde se ubica el área de estudio, están en situación de pobreza (CONEVAL, 2012).

Para el año 2010, en las comunidades ubicadas a la vera de la laguna Tecomate estaban asentados 844 habitantes: Tecomate Pesquerías tenía 68 \%, Las Ramaditas 18 \% y Cerro de la Pesquería 14 $\%$ de dicha población. Son localidades pequeñas que entre los años de 1970 y 2010, en su conjunto tuvieron un crecimiento neto de población de 53 habitantes. Destaca el caso de la comunidad de Cerro de la Pesquería, que tuvo un decrecimiento neto de 49 habitantes, aunque en 
su conjunto muestran un comportamiento poblacional propio de toda comunidad rural. Según INEGI (2011), la PEA ocupada en estas localidades estaba entre el rango de $32.89 \%$ a $34.21 \%$ con relación a la población total, y considerando que toda la PEA estaba ocupada, esto se puede tomar también como el rango del índice de dependencia económica; sin embargo, no se debe perder de vista que la PEA ocupada en las tres comunidades mayoritariamente desempeña actividades de autoempleo en el sector primario (agricultura y pesca).

El concepto de ingreso está muy relacionado con el de salario y puede entenderse de diferentes maneras: como un precio o como una entrada de capital, por ser un honorario por un trabajo prestado; así como un costo, dado que es una remuneración de un factor de producción. Sin embargo, el concepto de salario ha evolucionado con el progreso y hoy constituye uno de los problemas más complejos para el análisis de la organización económica y social de los pueblos. Los desequilibrios en salarios son capaces de provocar movilización social, porque los ingresos son dispares, y para la mayoría de la población son insuficientes y el empleo es escaso, propiciando el desarrollo de las actividades informales.

A lo largo del tiempo han surgido diferentes tesis alrededor de los salarios, cada una con sus aportaciones como: la teoría del Ingreso de Adam Smith, que considera los salarios dependientes de la riqueza. No obstante, el factor más importante se relaciona con el hecho de que la elevación de los salarios no necesariamente constituye un incremento en la cuantía de la riqueza nacional, el continuo aumento de esa riqueza depende del incremento de la producción y de la venta de la misma, ya sea en el interior del país o de su exportación (Tsoulfidis, 2010, p. 26).

Por otro lado, el ingreso desempeña un papel muy importante pues expresa cómo las familias definen sus estrategias de consumo a lo largo del tiempo, en la manera en que disponen su gasto (Torres y Gasca, 2001). También, ante situaciones de crisis desarrolla habilidades para contrarrestar efectos por la pérdida de ingresos tales como: usar más intensamente su fuerza de trabajo, incrementar la jornada laboral e incorporar nuevos miembros de la familia a las actividades diversas. A la vez, introduce cambios en la estructura de su gasto de bienes no básicos y básicos.

Los distintos niveles de pobreza rural y urbana se asocian con los bajos ingresos, lo que permite determinar cuáles grupos de la población, así como su ubicación, se encuentran en situación de vulnerabilidad, lo que se refleja en su nivel de bienestar. En el medio rural, la situación es preocupante ya que se menciona que en México $80 \%$ de la población no logra cubrir los mínimos 
alimentarios de acuerdo con las características de su ingreso; es población que desarrolla actividades de subsistencia en el sector primario (agricultura, pesca), o que ha migrado y se ubica en las áreas periféricas de las ciudades grandes y medias, sin contar con empleo fijo, viviendo en condiciones de hacinamiento y en ocasiones de la caridad pública.

En las localidades asentadas en los márgenes de la laguna de Tecomate, en promedio sólo $30 \%$ de la PEA ocupada percibe un salario, el resto no tiene empleo y desarrolla actividades de autoempleo o se traslada a San Marcos, la comunidad urbana más cercana, para ocuparse de manera temporal en actividades diversas. Algunos reciben apoyos económicos de los programas gubernamentales (empleo temporal, sesenta y más, madres solteras, jefa de familia), o de los migrantes que están en otras entidades o el extranjero. En estas localidades pesqueras, los ingresos están en función de la temporada de pesca y de los precios que asignan los intermediarios al producto de la captura. Sin duda, los ingresos de la población que se dedica a la pesca no son los más adecuados. Se requiere incentivar esta actividad para generar nuevas formas productivas y de organización de los pescadores, con el propósito de que diversifiquen sus actividades y puedan obtener ingresos complementarios que ayuden a paliar la pobreza en la que viven.

\section{Conclusiones}

En el caso de los habitantes de las localidades ubicadas a la vera de la laguna Tecomate, a pesar de que no demuestran intenciones y disposición para la organización colectiva en el desarrollo de las actividades pesqueras y agrícolas, debe pensarse en el desarrollo de propuestas institucionales que permitan mantener el equilibrio ecológico, promover actividades que tiendan a fortalecer la actividad pesquera, buscar que por medio del desfogue de la presa en el periodo de estiaje permita la alimentación de agua dulce y se propicie la reproducción de las especies, así como la diversificación de la actividad agrícola, puesto que uno de los mayores retos que enfrenta la región y el país es el de la producción de alimentos.

Por lo antes señalado, se requiere de la participación del gobierno federal, estatal y municipal, así como de las instancias de organización local, para implementar estrategias de desarrollo como señalan Rozas (2000) y Alburquerque (2004). Estas acciones de organización local permitirán de 
forma estratégica proponer iniciativas de desarrollo en la búsqueda de mejorar las condiciones de vida de la población asentada en el borde de la laguna de Tecomate.

\section{Bibliografía}

Alburquerque, F. (2004). Sistemas productivos locales: una mirada desde la política económica local para la generación de empleo, Seminario CEPAL-MTEySS, Buenos Aires. En línea. Recuperado el 22 de junio de 2016 de: http://www.trabajo.gov.ar/seminarios/files/09.pdf.

Black, R., Adger, W. N., Arnell, N. W., Dercon, S., Geddes, A., \& Thomas, D. (2011). The effect of environmental change on human migration. Global Environmental Change, 21(SUPPL. 1). En Línea. Recuperado el 31 de mayo de 2016 de: http://dx.doi.org/10.1016/j.gloenvcha.2011.10.001

Bayley, B. y Petrere, M. (1986). Amazon fisheries and the aquatic system: current status. Documento presentado al Simposio LARS (International Large River Symposium), 21 de septiembre de 1986. Ontario.

Cassels, Susan., Curran, Sara R. \& Kramer, Randall (2005). Do Migrants degrade Coastal Environments? Migration, Natural Resource Extraction and Poverty in North Sulawesi, Indonesia. Human Ecology, vol. 33, núm. 3. En Línea. Recuperado el 26 de Abril de 2016 de: DOI: 10.1007/s10745-005-4142-9.

Consejo Nacional de Evaluación de la Política de Desarrollo Social (Coneval). Informe de pobreza y evaluación en el estado de Guerrero 2012. Consejo Nacional de Evaluación de la Política de Desarrollo Social. México, D.F, 56 pp.

García-Zamora, R., O. Pérez-Veyna., G. Foladori., R. Delgado-Wise., M. Moctezuma- Longoria., E. Reyes-Rivas., Humberto. Márquez-Covarrubias., P. Rivera-Castañeda (2007). Paradojas de la migración internacional y el medio ambiente. Economía Sociedad y Territorio, vol. VI, núm. 24, 975-994. El Colegio Mexiquense, A.C. 
INEGI (1983). X Censo General del Población y Vivienda, 1980. Estado de Guerrero, Instituto Nacional de Estadística, Geografía e Informática, Aguascalientes, México.

INEGI (1991). XI Censo General del Población y Vivienda, 1990. Estado de Guerrero, Instituto Nacional de Estadística, Geografía e Informática, Aguascalientes, México.

INEGI (2001). XII Censo General de Población y Vivienda 2000, Estado de Guerrero, Instituto Nacional de Estadística, Geografía e Informática, Aguascalientes, México.

INEGI (2011). XIII Censo General de Población y Vivienda 2010, Estado de Guerrero, Instituto Nacional de Estadística, Geografía e Informática, Aguascalientes, México.

INEGI (30 agosto de 2016). Marco geoestadístico nacional. Recuperado de: http://www3.inegi.org.mx/sistemas/biblioteca/ficha.aspx?upc=702825004386

Madera, J. A. (2010). Tabaco y migración: entre la reproducción social y la sobrevivencia, en De Aquí, De Allá. Migración y Desarrollo Local, Eduardo Meza Ramos y Lourdes C. Pacheco Ladrón de Guevara (Coordinadores), Universidad Autónoma de Nayarit, $1^{\text {a }}$ edición.

Novoa, F. (1986). The multispecies fisheries of the Orinoco River: Development, present status and management strategies. Documento presentado al Simposio LARS (International Large River Symposium), 21 de septiembre de 1986. Ontario.

Rozas, G. (2000). Pobreza y desarrollo local, Universidad de Chile. En Línea. Recuperado el 29 de junio de 2016 de: http://www.redelaldia.org/IMG/pdf/0386.pdf

Secretaría de Industria y Comercio (1973). IX Censo de Población 1970, Estado de Guerrero. Dirección General de Estadística, México. 
SEMARNAT (s.f.). Recuperado el 07 de septiembre de 2016 de: http://sinat.semarnat.gob.mx/dgiraDocs/documentos/gro/estudios/2008/12GE2008E0030. pdf

Torres T, F. y Gasca Z, J. (2001). Ingresos y alimentación de la población en el México del siglo XX. Instituto de Investigaciones Económicas-UNAM, México.

Tsoulfidis, L. (2010). Competing Schools of Economic Thought, Springer. En Línea. Recuperado el 27 de julio de 2016 de: http://link.springer.com.etechconricyt.idm.oclc.org/chapter/10.1007/978-3-540-92693-1_4

Villaseñor, A. y García, R. (1990). La Universidad de Guadalajara y la pesca en Jalisco. Informe interno, Universidad de Guadalajara, México. 74 pp.

Villerías-Salinas, S., Violante-González, J., García-Castro, N. and Alonzo-Guzmán, L. (2016). Environmental Deterioration of the Tecomate Coastal Lagoon, in the Guerrero State, Mexico. International Journal of Geosciences, 7, 1-10. En Línea. Recuperado el 30 de mayo de 2016 en: doi: 10.4236/ijg.2016.71001. 